\title{
Psychologie der Kreativität
}

\author{
VON JOACHIM FUNKE
}

Ein kleiner Streifzug durch die psychologische Kreativitätsforschung befasst sich mit den Möglichkeiten der Erfassung kreativer Prozesse, ihrer Manifestation, den Determinanten, der Frage nach der Notwendigkeit zu kreativem Denken und schließlich Erkenntnissen darüber, wie kreatives Denken gefördert werden kann.

\section{Einleitung}

Kreatives Denken - wirft nicht bereits der kurze Titel eine Reihe von Fragen auf? Muß nicht Denken immer etwas Neues hervorbringen, kann Denken überhaupt ohne Kreativität gedacht werden? Aber auch: ist ein kreativer Prozess überhaupt der Ratio, dem Denken zugänglich, oder spielen hier nicht vielmehr irrationale Elemente eine viel wichtigere Rolle? Sind diese Prozesse überhaupt mit den wissenschaftlichen Methoden der Psychologie erfassbar? Besitzt jeder Mensch ein kreatives Potenzial? Mit dem vorliegenden Beitrag soll diesen Fragen nachgegangen werden, indem wir zunächst einmal anstelle einer Antwort weitere Fragen stellen:

1. Welche Untersuchungsmöglichkeiten stehen der Kreativitätsforschung zur Verfügung? Woher stammt überhaupt unser Wissen über das Thema?

2. Wie sieht kreatives Denken aus, wie manifestiert es sich?

3. Was sind Determinanten kreativen Denkens?

4. Warum brauchen wir kreatives Denken in unserer heutigen Zeit mehr denn je?

5. Was kann man tun, um kreatives Denken zu fördern?

Die Behandlung dieser Fragen kann hier sicher nicht erschöpfend vorgenommen werden, aber die geneigte Leserin und der geneigte Leser dürfen doch erwarten, nach Lektüre dieses Beitrags den Fragen nicht mehr ratlos gegenüberzustehen. 


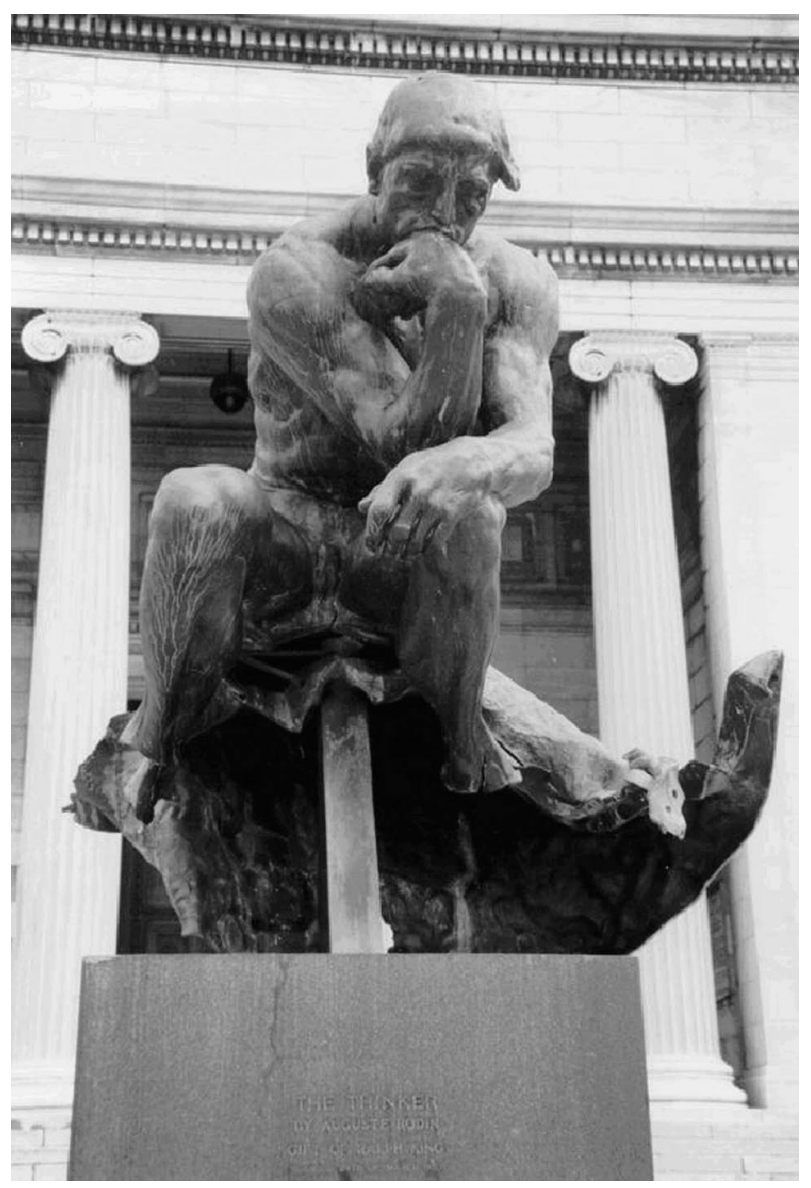

Abb. 1. Der Denker von Rodin [1]

Wie Simonton (2000) ausführt, ist Kreativität eine allgegenwärtige Erscheinung menschlicher Natur: Das Gebäude, in dem Sie sich möglicherweise befinden, ist von einem Architekten gestaltet; die Kleidung, die Sie tragen, wurde von einem Designer entworfen; der Sessel, auf dem Sie sitzen, wurde - hoffentlich ergonomisch korrekt - gestaltet; das Buch, das Sie lesen, wurde entworfen und gestaltet; usw. Hinter all den Dingen um Sie herum, die wir gemeinhin Artefakte nennen, steht eine Person, die diese Dinge kreiert und damit eine ganz bestimmte Absicht verfolgt hat. Diese Allgegenwart kreativer Akte steht in krassem Missverhältnis zu ihrer Erforschung. Über lange Zeit hinweg hielt man nämlich kreative Akte für etwas, das wie ein Blitz über die Person kommt und keine weitere Erklärung erlaubte. Erst mit dem Beginn der naturwissenschaftlichen Psychologie Ende des 19. Jahrhunderts sollten sich diese Ansichten langsam ändern. 
Letztlich ist es wohl erst einem vehementen Plädoyer für mehr Kreativitätsforschung von John P. Guilford (1950) zu verdanken, dass tatsächlich ein fulminanter Zuwachs an Erkenntnissen in den 6oer und 7oer Jahren des 20. Jahrhunderts erfolgte. Doch so wichtig Guilfords Aufruf an die „scientific community" war: mindestens ebenso bedeutsam war ein Ereignis in einer völlig anderen Disziplin - der Raumfahrt. Historisch gesehen hat die Kreativitätsforschung einen erheblichen Schub bekommen durch den sog. „Sputnik-Schock“ der Amerikaner (so auch Amelang \& Bartussek 1997, S. 260). Am 4. Oktober 1957 startete ein kleiner Satellit vom russischen Raketenbahnhof Baikonur und umrundete die Atmosphäre - das Selbstverständnis der Amerikaner als die technologisch führende Weltmacht wurde dadurch in seinen Grundfesten erschüttert.

Aus Sorge, nicht genug originelle Wissenschaftler zu produzieren, wurden sofort großangelegte Programme gestartet (Stichwort: „Headstart”), mit denen begabte Menschen identifiziert und gefördert werden sollten. Im Zuge dieser Forschungen sind eine Menge neuer Erkenntnisse über kreative Prozesse gewonnen worden, die unser Bild nachhaltig geprägt haben. Bevor hierauf näher eingegangen werden wird, soll zunächst die Frage nach den wissenschaftlichen Untersuchungsmöglichkeiten gestellt und beantwortet werden.

\section{Welche Untersuchungsmöglichkeiten stehen zur Verfügung?}

Eine psychometrisch exakte Erfassung der Kreativität einer Person stellt ein schwieriges Unterfangen dar. Dennoch haben sich eine Reihe von Psychologen dieser Herausforderung gestellt. ${ }^{1}$ Generell ist zwischen sprachlichen und sprachfreien Verfahren zu unterscheiden.

Die sprachlichen Verfahren verlangen bestimmte Leistungen, die sich in sprachlichen Äußerungen niederschlagen. So ist etwa aus Guilfords Konzept des divergenten Denkens (mehr dazu weiter unten) ein Test unter dem Namen „Unusual Uses“ hervorgegangen, bei dem möglichst viele verschiedene Verwendungszwecke für einen bestimmten Gegenstand zu benennen sind. Sie kennen sicher den berühmten Ziegelstein, dem man nicht zur zum Bauen von Häusern oder Regalen nutzen kann, sondern auch als Bettwärmer, Kofferraumbeschwerer oder Wurfgeschoss. Die Flexibilität des Denkens äußert sich nicht nur in der reinen Quantität der Nennungen, sondern auch in der Anzahl verwendeter Kategorien wie Baumaterial, Speichermedium oder Waffe. Neben Flexibilität und Flüssigkeit interessiert aber auch die Originalität von Antworten. Dass man einen Ziegelstein als Schwamm benutzen

\footnotetext{
${ }^{1}$ Eine umfassendere Übersicht über Testverfahren zur Messung der Kreativität gibt Krampen (1993).
} 
kann, fällt eben nicht jedem ein und ist daher origineller als dessen Verwendung zum Bauen.

Bei einem anderen sprachlichen Kreativitätstest, der von Mednick (1962) unter dem Namen „Remote Associates Test" vorgeschlagen wurde, ist das Gemeinsame von drei nur gering assoziierten Reizwörtern zu finden. Das Verbindende etwa von „Humor“, „Pech“ und „Nacht“ ist die Schwärze. Gemessen wird hier die Flexibilität von Assoziationen.

Natürlich kann man fragen, ob diese Verfahren wirklich etwas über Kreativität aussagen. In erster Linie wird mit den genannten Verfahren die Verfügbarkeit begrifflicher Strukturen im semantischen Gedächtnis geprüft - sicher nicht unwichtig für kreative Prozesse, aber eben auch nicht erschöpfend.

Die sprachfreien Verfahren zur Erfassung von Kreativität beruhen im wesentlichen auf zeichnerischen Aktivitäten, die von der Testperson verlangt werden. So sollen im „Torrance Test of Creative Thinking“ (Torrance 1966) vorgegebene Bilder entweder vervollständigt, neu kombiniert oder produziert werden. Abbildung 1 enthält ein Beispiel für jede der drei Aufgaben.

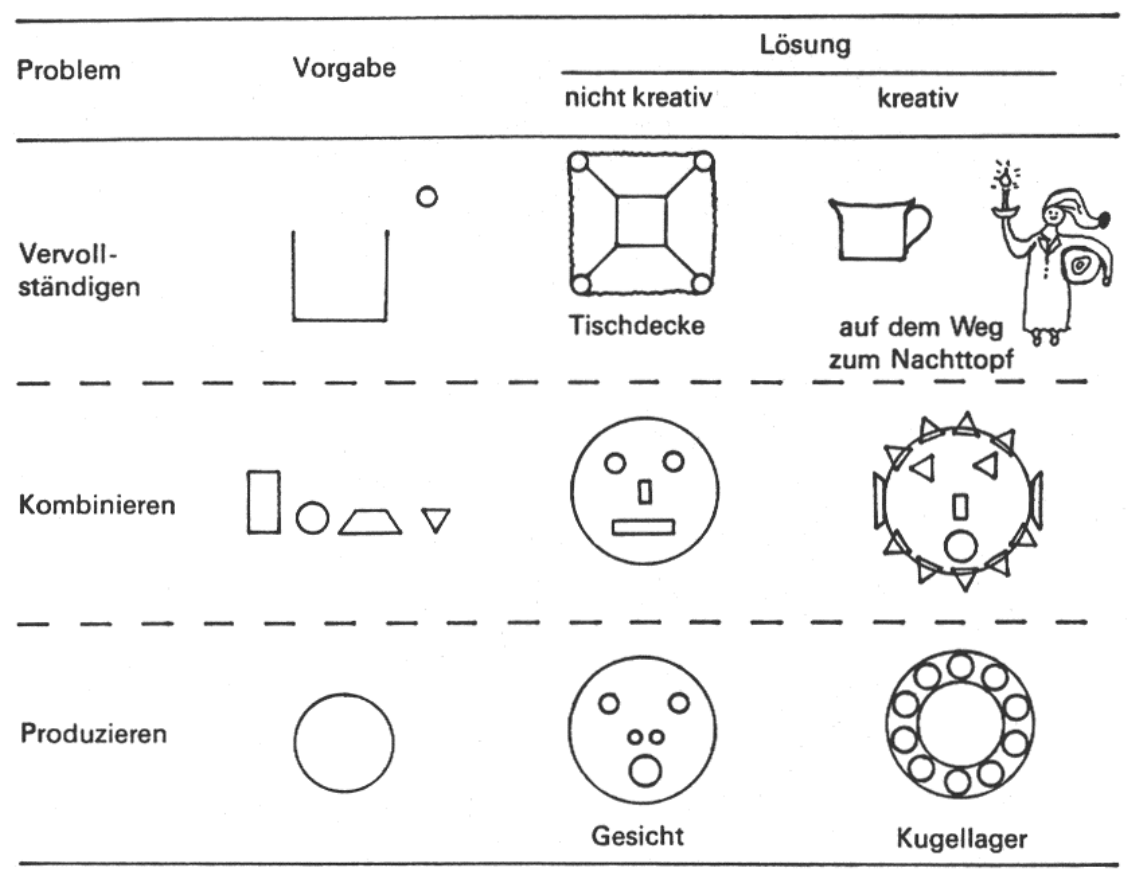

Abb. 2. Beispiel für sprachfreie Aufgaben aus dem „Torrance Test of Creative Thinking“ [2] 
Einer kritischen Betrachtung halten sowohl die sprachlichen wie auch die sprachfreien Verfahren kaum stand. Hussy (1986, S. 78) geht sogar so weit festzustellen, „dass sie [die Messverfahren] zur Erfassung des kreativen Prozesses als untauglich zu bezeichnen sind“.

Auch wenn die psychometrische Erfassung der Kreativität in Form eines zuverlässigen und standardisierten Diagnostikums damit entfällt, bleiben natürlich experimentelle Verfahren, über die kurz zu berichten ist.

Wichtige Erkenntnisse auf der Basis experimenteller Studien kommen aus dem Bereich analogen Transfers (vgl. Gentner \& Gentner 1983; Holyoak 1985). Hier geht es vor allem um die Frage, ob Personen spontan oder auch mit Hilfen die strukturelle Ähnlichkeit zweier verschiedener Gebiete erkennen, etwa die Analogie zwischen dem Sonnensystem und dem Atomsystem, gegeben durch die Tatsache, dass in beiden Systemen einzelne Elemente auf einer Kreisbahn um einen Kern rotieren und von diesem angezogen werden. Natürlich handelt es sich auch hierbei nur um einen Aspekt von Kreativität.

Die Ergebnisse zum analogen Transfer zeigen vor allem die Schwierigkeit von Personen, die tiefenstrukturellen Parallelen zweier an der Oberfläche unterschiedlicher Domänen überhaupt spontan zu erkennen. Wird man dagegen auf die Ähnlichkeit hingewiesen, lassen sich die Eigenschaften der Ausgangsdomäne oft nutzbringend auf die neue Zieldomäne übertragen, etwa indem Lösungsstrategien der einen Domäne auf die andere übertragen werden.

Im Kontext wissenschaftlicher Entdeckungen steht das bei Analogieschlüssen praktizierte Induktionsprinzip natürlich erkenntnistheoretisch in Widerspruch zum Prinzip der Falsifikation. Dem entdeckenden Wissenschaftler wird hier abverlangt, einerseits zur kreativen Generierung von Hypothesen das Induktionsprinzip zu nutzen, um aus möglichst vielen Analogien Erkenntnisgewinn zu ziehen, andererseits aber dem deduktivistischen Falsifikationsprinzip zu folgen, bei dem es um die strenge Prüfung von Hypothesen geht (Bredenkamp 1980).

Neben diesen Untersuchungsmöglichkeiten aus dem Bereich analogen Transfers und den bereits genannten Testverfahren stehen Kreativitätsforschern weitere Verfahren zur Verfügung. Hocevar und Bachelor (1989) nennen in ihrem Übersichtsbeitrag zusätzlich folgende Meßmethoden: (1) Persönlichkeitsinventare, mit denen bestimmte Eigenschaften erfasst werden können; (2) biographische Inventare, die Hintergrund-Informationen zu einer Person und ihren Entwicklungsbedingungen einholen; ${ }^{2}$ (3) Einstellungsund Interessen-Skalen, die bestimmte Vorlieben sichtbar machen; (4) personenbezogene Einschätzungen von Lehrern, „peers“ und Vorgesetzten, die

\footnotetext{
${ }^{2}$ Die Bedeutung biographischer Analysen als Quelle der Kreativitätsforschung betont z.B. Gardner (1993).
} 
die zu beurteilende Person über längere Zeit hinweg beobachtet haben und sie kennen; (5) Eminenz-Einschätzungen (z.B. Zitierhäufigkeit, eingeräumter Platz in Biographien, Preise); (6) Selbstberichte über besondere Leistungen anhand von Checklisten; (7) Beurteilungen kreativer Arbeitsproben und Produkte.

Für jede einzelne Methode gibt es Vor- und Nachteile, so dass die Antwort auf die Frage nach den Untersuchungsmöglichkeiten sehr differenziert ausfällt. Auch wenn es nicht „das“ Maß für Kreativität gibt: aus der Zusammenschau verschiedener derartiger Zugänge kann doch ein zuverlässiges Bild entstehen. Wie dieses Bild aussieht, wird in den nächsten Abschnitten deutlicher.

\section{Wie sieht kreatives Denken aus?}

Im Unterschied zur bereits erwähnten populären Vorstellung, wonach kreative Denkakte Ergebnisse eines Geistesblitzes seien, also unmittelbar als Lösung eines Problems vor dem geistigen Auge erschienen (Boden 1991), geht die psychologische Forschung bereits seit den frühen Arbeiten von Wallas (1926) davon aus, dass die kreative Lösung Ergebnis eines langdauernden, oft sogar mehrjährigen Prozesses ist (siehe auch Weisberg 1989). Mindestens fünf Stufen des kreativen Prozesses werden traditionell unterschieden, die hier kurz genannt sein sollen.

Stufe 1: Vorbereitung. Es ist schwierig eine gute Idee zu bekommen, ohne sich nicht vorher intensiv mit dem fraglichen Gebiet beschäftigt zu haben. Kreative Erfinder kennen die wichtigsten Prinzipien in ihrer Disziplin, kreative Künstler haben sich intensiv mit den Werken von Vorgängern und Zeitgenossen auseinandergesetzt, kreative Wissenschaftler haben nicht nur ein langes Studium hinter sich, sondern auch zugleich einen hohen Grad an Expertise in ihrem Fach erreicht („exceptional talents are less born than made“, vgl. Ericsson 1996). Intensive Vorbereitung ist also nötig. ${ }^{3}$

Stufe 2: Inkubation. Interessanterweise kann es sinnvoll sein, ein Problem, für das man eine kreative Lösung sucht, einfach liegen zu lassen. In den Phasen der Nichtbeschäftigung arbeitet unser Gehirn offensichtlich weiter - die Inkubationsphase tritt in Kraft, nachdem die vorangegangene Phase die Grundlagen durch eine Art „gedanklicher Infektion“ dafür gelegt hat.

Was in dieser Inkubationsphase genau passiert, war lange Zeit unklar. Am Werk ist hier die Dynamik unseres Gedächtnisses, in dem assoziative Verbindungen zwischen Ideen und Vorstellungen sich im Laufe der Zeit ab-

\footnotetext{
${ }^{3}$ Unter Expertise-Forschenden (z.B. Ericsson 1996) wird davon gesprochen, dass, grob gesagt, von Expertise gesprochen werden kann ab einer Beschäftigungszeit von 10 ooo Stunden mit einem bestimmten Thema.
} 
schwächen und durch neu hinzukommende Informationen überlagert und verändert werden („creative cognition“, vgl. Finke, Ward \& Smith 1992). Die in der Inkubationsphase ablaufenden Prozesse bleiben der kreativen Person unbewusst und können nicht aktiv beeinflusst werden. Allerdings hat gerade die neuere Forschung zum Kognitiven Unbewussten eindrucksvolle experimentelle Belege intuitiver Informationsverarbeitung vorgelegt, die diese Phase entmystifizieren (siehe z.B. Dorfman, Shames \& Kihlstrom 1996, sowie die Beiträge bei Smith, Ward \& Finke 1995 und Ward, Smith \& Vaid 1997).

Stufe 3: Einsicht. Zu einem ungewissen Zeitpunkt durchdringt eine rekombinierte Assoziation die Schwelle zum Bewusstsein und liefert den Moment der Erleuchtung - die Illumination. Gestaltpsychologen haben vom „Aha“Effekt gesprochen. Dies ist der Moment der Bewusstwerdung des schöpferischen Augenblicks, der nach entsprechender Vorbereitung und daran anschließender Inkubation erfolgt; in medizinischer Terminologie haben wir die „Krisis“ erreicht.

Stufe 4: Bewertung. Die in der Erleuchtungsphase gewonnene kreative Einsicht muss natürlich bewertet werden - nicht alle kreativen Einsichten sind wirklich brauchbar. Hier kommen Normen und Werte ins Spiel, die darüber entscheiden, ob eine neue Idee der kritischen Zensur zum Opfer fällt oder es schafft, diese Hürde zu überwinden. Dabei ist die nächste Stufe nicht unbeteiligt.

Stufe 5: Ausarbeitung. Von der ersten Idee einer elektrischen Glühbirne bis zum ersten Prototypen war ein weiter Weg zu überwinden. Thomas Edison hat einmal rückblickend gesagt: „Genie bedeutet $1 \%$ Inspiration und $99 \%$ Transpiration“, womit er auf die Kräfte hinweist, die zur Durchsetzung einer kreativen Idee nötig sind. Außerdem können sich auf dem Weg von der ersten Idee hin zum fertigen Endergebnis - einem Bild, einem technischen Produkt, einem Roman - noch zahlreiche Überraschungen und Änderungen ergeben.

Soviel also zunächst einmal zu den fünf Stufen eines kreativen Prozesses, die sich nach heutiger Sicht als völlig normale kognitive Verarbeitungsprozesse darstellen. Auf die Frage „Wie sieht kreatives Denken aus?“ haben wir damit eine erste Antwort gegeben, die ich noch um eine Facette ergänzen möchte. Hierbei handelt es sich um die von Guilford eingeführte Unterscheidung von konvergentem und divergentem Denken (siehe zusammenfassend Guilford 1967).

Unter konvergentem Denken versteht Guilford logisches Vorgehen, das auf einen ganz bestimmten Lösungspunkt hinführt. Unter divergentem Denken, das bei kreativen Prozessen vorherrscht, geht es dagegen um unübliche Assoziationen, um den Wechsel von Perspektiven, um die Verbreiterung des 


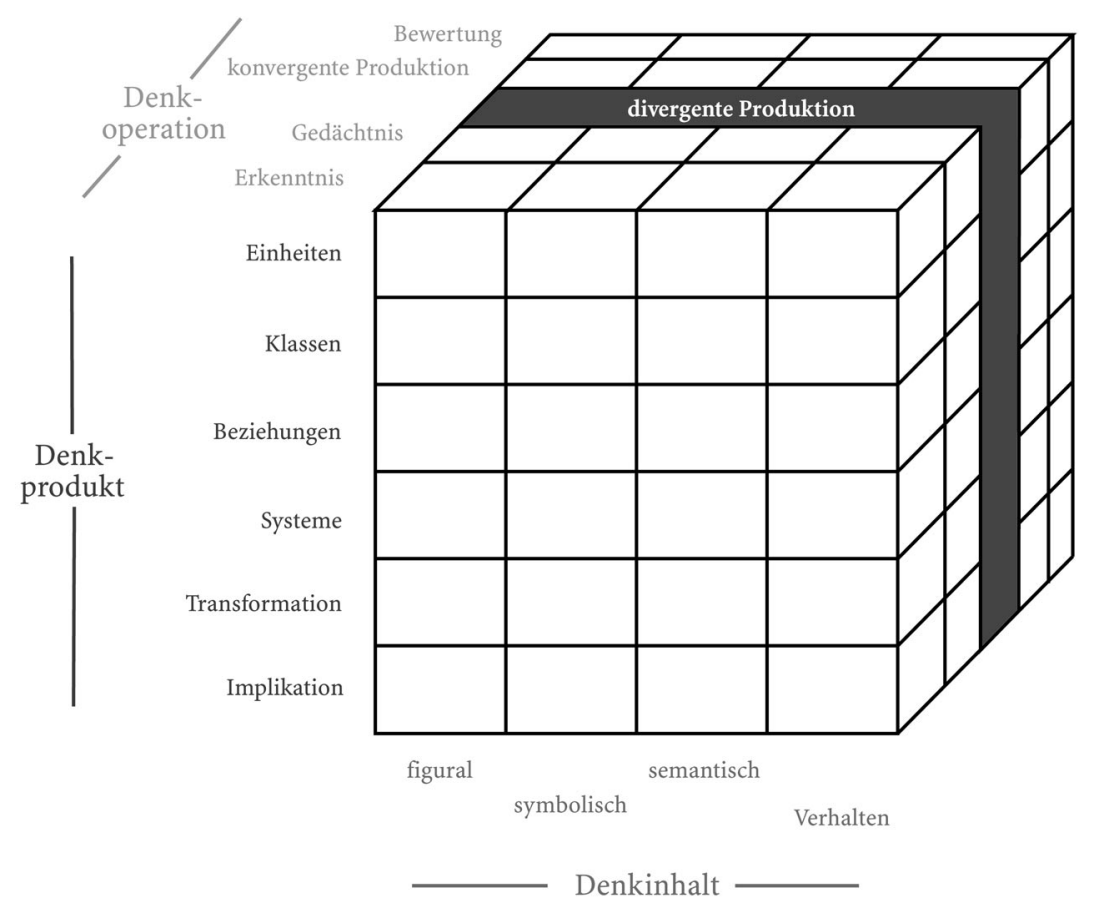

Abb. 3. Würfelmodell der Intelligenz nach Guilford: 5 Denkoperationen, die in 4 Denkinhalten ablaufen können und 6 Denkprodukte erzeugen können. Jeder der 120 Würfelzellen verkörpert eine der Kombinationen. Hervorgehobene ist die für Kreativität zentrale Operation der divergenten Produktion. [3]

Horizonts. Normalerweise beginnt ein Problemlöseprozess damit, dass zunächst eine größere Menge an Ideen generiert wird (= divergent), aus denen dann eine oder mehrere für die vertiefende Ausarbeitung ausgewählt werden (= konvergent).

Eine weitere begriffliche Unterscheidung betrifft produktives versus reproduktives Denken. Auch wenn eine Abgrenzung nicht scharf vollzogen werden kann: als Ankerpunkt einer Dimension lassen sich die beiden Prozesse präziser beschreiben. Unter reproduktivem Denken werden solche kognitiven Prozesse verstanden, die zur Lösung eines Problems (genauer: einer Aufgabe) einfach nur abgerufen werden müssen. Als Beispiel könnte man das Multiplizieren zweier Zahlen durch eine rechenkundige Person betrachten: auch wenn sie das Produkt aus 369 und 246 vielleicht noch nie berechnet hat, das Ergebnis für sie also neu ist, ist doch der Rechenweg (die Anwendung des entsprechenden „Operators“) bekannt. Ganz anders beim produktiven Denken, bei dem der Lösungsweg neu konstruiert werden muss. Produktives, kreatives Denken soll Lösungsmöglichkeiten für ein Problem erst einmal erzeugen, womit die Frage nach der Abgrenzbarkeit von kreati- 
vem Denken und Problemlösen aufgeworfen wird. Tatsächlich liegen beide Konstrukte nah beieinander, und insbesondere beim Lösen komplexer Probleme (vgl. Funke 1999) sind kreative Lösungen unabdingbar.

Doch was beeinflusst kreative Prozesse nun genau? Auf die sog „Determinanten“ kreativen Denkens geht der nächste Abschnitt ausführlicher ein.

\section{Was sind Determinanten kreativen Denkens?}

Klassischerweise werden verschiedene Perspektiven der Kreativitätsforschung unterschieden: die kreative Person, der kreative Prozess und das kreative Produkt. Nachdem im vorangehenden Teil bereits einige Aussagen zum kreativen Prozess gemacht wurden, soll hier der Schwerpunkt auf die kreative Person und ihr Umfeld gelegt werden. Auch ein paar Bemerkungen zum kreativen Produkt sollen hier erfolgen.

\section{Merkmale der Person}

Muss eigentlich eine überdurchschnittliche Intelligenz gegeben sein, um kreative Leistungen zu erbringen? Diese Frage haben bereits Galton (1869) aus der Perspektive der Vererbungsforschung und Terman (1925, vgl. auch Subotnik \& Arnold 1994) aus der Perspektive der Hochbegabtenforschung gestellt und durch empirische Studien zu beantworten versucht. Sternberg (1995, S. 366) bejaht diese Frage, schränkt aber zugleich ein: „bright but not brilliant", womit zum Ausdruck gebracht wird, dass oberhalb einer gewissen Intelligenzschwelle ${ }^{4}$ eine Erhöhung der Intelligenz keine Auswirkungen mehr bezüglich kreativer Leistungen bewirkt. Dabei sollte man sich allerdings eine Intelligenzkonzeption vor Augen halten, die nicht von der Annahme einer einzelnen "generellen“ Intelligenz ausgeht, sondern die - wie dies etwa in den Vorstellungen von Howard Gardner (1983) zum Ausdruck kommt - die Existenz "multipler Intelligenzen“ annimmt (sprachliche, logisch-mathematische, räumliche, musikalische, motorische sowie personale Intelligenz).

Aber nicht nur Intelligenz interessiert an dieser Stelle - vielmehr wird die Frage allgemeiner gestellt: gibt es spezifische Ausprägungen von Persönlichkeitseigenschaften kreativer Personen? Die heutigen Ergebnisse (z.B. Martindale 1989; Simonton 1999) zeichnen ein Profil, in dem Unabhängigkeit, Nonkonformismus, unkonventionelles Verhalten, weitgespannte Interessen, Offenheit für neue Erfahrungen, Risikobereitschaft sowie kognitive und verhaltensmäßige Flexibilität als Kennzeichen dienen. Auch die alte Debatte um Genie und Wahnsinn findet insofern Unterstützung, als Kreativität tatsächlich oftmals mit einem gewissen Grad psychopathologischen Verhaltens einhergeht (vgl. Eysenck 1995; Ludwig 1995). Allerdings sind pathologische

\footnotetext{
${ }^{4}$ Getzels und Jackson (1962) haben die Schwelle bei einem IQ von 120 angesetzt.
} 
Verhaltensweisen keine notwendige Voraussetzung für Kreativität - im Gegenteil: oftmals beweist gerade die kreative Persönlichkeit, wie bestimmte psychologische Schwachpunkte in einem adaptiven Sinn nützlich gemacht werden können (siehe Csikszentmihalyi 1997; Ludwig 1995; Rothenberg 1990).

In Hinblick auf das Lebensalter wird oft argumentiert, dass die Kreativität nach einem Höhepunkt zwischen 20 und 30 mit zunehmendem Alter nachlasse (z.B. Lindauer 1993). Tatsächlich ist eine derart pessimistische Aussage allerdings wohl nicht gerechtfertigt, da zahlreiche Faktoren für eine qualitative wie quantitative Wiederbelebung kreativer Produktivität im späteren Lebensalter sorgen können (siehe Csikszentmihalyi 1997; Simonton 1997). - Über Geschlechtsunterschiede im Kontext der Kreativität informiert der Beitrag von Annette Kämmerer (in diesem Band).

\section{Das kreative Umfeld}

Forschung, die die gesamte Lebensspanne übergreift, zeigt interessanterweise, dass Kreativität nicht immer dort entsteht, wo die besten Bedingungen vorliegen - eher im Gegenteil scheinen herausfordernde Erfahrungen die Fähigkeiten einer Person zu stärken, Widerständen zu begegnen (Simonton 1994). Dies macht deutlich, dass nicht die kreative Person allein maßgeblich ist, sondern dem kreativen Umfeld eine große Bedeutung zukommt. Dieses „Feld“ besteht aus anderen Personen, die kreativ auf dem gleichen Gebiet tätig sind. Martindale (1990) etwa macht deutlich, dass für einen Schriftsteller hauptsächlich andere Schriftsteller (sowie einige ausgewählte Kritiker) als Referenz gelten - diese Strukturen findet Martindale auch in der Kunst und in der Musik. Diese Überlegungen machen deutlich, dass nicht die alleinige Konzentration auf eine einzelne kreative Person ausreicht, um das Zustandekommen eines kreativen Produkts zu verstehen.

Neben dem eben beschriebenen Einfluß des „Feldes“ gehören natürlich auch soziokulturelle Einflüsse („Zeitgeist“) zum kreativen Umfeld. So haben im geschichtlichen Verlauf viele Länder nach der Gewinnung ihrer Unabhängigkeit kreative Blütezeiten durchlebt, angefangen mit dem antiken Griechenland. Wie Simonton (1994) ausführt, mag dies mit Tendenzen zusammenhängen, die zu mehr Heterogenität anstatt Homogenität ermutigen. Kulturelle Diversität wird dort sogar als kreativitätsförderlicher Faktor gesehen. Historiometrische Analysen kreativer Produkte (Simonton 1984) scheinen diese Sicht zu bestätigen.

\section{Das kreative Produkt}

In Hinblick auf das kreative Produkt, das Ergebnis kreativen Denkens, werden zwei Kriterien als zentral erachtet, nämlich (a) Neuigkeit und (b) 
Angemessenheit und Nützlichkeit im Sinne einer Problemlösung. Natürlich hängt die wahrgenommene Neuigkeit vom Hintergrund der beurteilenden Person wie auch vom sozialen Konsens ab, und selbstverständlich kann eine von mir vorgenommene Entdeckung durchaus Neuigkeitswert beanspruchen, auch wenn ich später erfahre, dass es sich um eine längst gemachte Entdeckung handelte.

Das an zweiter Stelle genannte Kriterium der Angemessenheit und Nützlichkeit soll sicherstellen, dass nicht alles, was neu ist, auch automatisch als kreativ angesehen wird. Vielmehr sollen bestimmte Einschränkungen, die das Problem vorgibt (z.B. bei der Beleuchtung dunkler Innenräume), möglichst optimal getroffen werden. Großflächige Spiegelsysteme wären im Regelfall dafür ungeeignet.

Neben den beiden Hauptkriterien werden von Lubart (1994, S. 291) noch drei Nebenkriterien angeführt: (c) Qualität, (d) Bedeutung und (e) Entstehungsgeschichte. Mit diesen Zusatzkriterien kann die Bewertung eines Produkts als kreativ verändert werden. In Hinblick auf Qualität dürfte nachvollziehbar sein, dass ein qualitativ hochstehendes neues Produkt einem noch unausgereiften Produkt überlegen ist. Die Bedeutung eines Produkts ergibt sich aus dessen Reichweite: eine neuartige Alarmanlage für Autos, bei der Tierberührungen keinen Fehlalarm auslösen, hat eine geringere Reichweite als eine neuartige Methode, Sonnenenergie zum Kochen zu verwenden. Die Entstehungsgeschichte kann die Bewertung insofern verändern, als wir bei Kenntnis einer rein zufälligen Entdeckung weniger Respekt vor der kreativen Leistung haben, als wenn wir von der mühevollen, langjährigen Entwicklungsarbeit an einem Produkt hören.

Dass Urteile bezüglich des kreativen Werts eines Produkts nicht nur vom historischen Kontext, sondern auch von der sozialen Bezugsgruppe abhängig sind, merkt man an der großen Spannbreite von Beurteilungen bei ein und demselben Produkt. Dies liegt - so Lubart (1994) - zum einen am bereits erwähnten unterschiedlichen Hintergrund der Beurteiler. Kunstlehrer, die viele Zeichnungen gesehen haben, beurteilen das Bild eines Kindes kritischer als die Eltern, die von seinen ersten Produkten ganz begeistert sind, aber kaum Vergleichsmöglichkeiten haben. Zum anderen liegt es auch an der unterschiedlichen Gewichtung der hier aufgeführten Kriterien. Je nach deren anteiliger Bedeutung für das Gesamturteil lassen sich unterschiedliche Bewertungen dadurch gut erklären.

\section{Warum brauchen wir kreatives Denken?}

Die Notwendigkeit kreativen Denkens für den Fortbestand unserer Welt dürfte außer Zweifel stehen, auch wenn es gerade kreative Erfindungen waren, die die Menschheit mit der Möglichkeit ihrer Selbstzerstörung konfron- 
tiert haben. Brauchen ausgewiesene Experten und Expertinnen in einem Fach überhaupt Nachhilfe in Sachen Kreativität? Interessanterweise ja, denn gerade Fachwissen kann gegenüber neuen Ideen blind machen („deformation professionelle").

Bereits 1942 hat Luchins anhand seiner Wasser-Umschüttprobleme experimentell demonstriert, wie schnell Menschen bestimmte Strategien aufbauen und später selbst dann routinemäßig anwenden, wenn es einfachere Wege zur Lösung gibt. Seine Versuchspersonen sollten eine bestimmte Menge Wasser durch Verwendung unterschiedlich großer Messbecher abmessen. Die durch wiederholte Anwendung einer Prozedur entstehende Automatisierung (z.B. „zweimal den großen Messbecher hineinschütten, dann einmal mit dem kleinen Messbecher abschöpfen") macht unempfindlich für alternative, einfachere Vorgehensweisen, die zu einem späteren Versuchszeitpunkt zugelassen werden. Die Gestaltpsychologen sprachen in diesem Kontext von „funktionaler Gebundenheit" und von „Einstellungseffekten“.

Peter Frensch und Bob Sternberg haben 1989 ein interessantes Experiment durchgeführt, bei dem sie Bridge-Spieler mit unterschiedlichen Spielstärken gegen kontrollierte Computergegner antreten ließen. Die Hälfte der Spiele fand unter normalen Spielbedingungen statt, die andere Hälfte wurde mit neuen, modifizierten Spielregeln gespielt, die entweder nur OberflächenMerkmale der Regeln oder aber deren Tiefenstruktur betrafen. Dabei zeigte sich, dass die Experten stärker als die Novizen unter der tiefen Regeländerung litten, aber selbst dort noch besser und schneller als die Anfänger abschnitten. Dennoch zeigen diese Ergebnisse, dass Experten Schwierigkeiten in der Anpassung ihres Wissens an neue Bedingungen aufweisen. Manchmal kann es eben auch ein Nachteil sein, wenn man mehr weiß.

Josef Krems (1995) beschreibt eine Serie von Experimenten, in denen Novizen wie Experten eines Gebietes (Internisten, Automechaniker, Programmierer) Hypothesen bilden mussten und Schlussfolgerungen aus bestimmten, vorgegebenen Symptomatiken ziehen sollten. Über alle von ihm untersuchten Gegenstandsbereiche hinweg konsistent zeigen sich folgende Befunde: (1) Experten modifizieren ihre Hypothesen bei der Ursachensuche häufiger als Novizen, (2) Experten unterliegen in geringerem Maß als Novizen einer Bestätigungstendenz (d.h. der stärkeren Beachtung bestätigender und der geringeren Beachtung falsifizierender Informationen), und (3) die Fähigkeit zum flexiblen Hypothesenwechsel basiert mehr auf fallbasiertem Wissen (im Unterschied zu Regelwissen) und bleibt damit an bestimmte Gegenstandsbereiche und die darin gemachten Erfahrungen gebunden.

Nach diesen Befunden steht es um die Flexibilität von Experten doch nicht so schlecht, wie die experimentelle Studie von Frensch und Sternberg (1989) befürchten ließ. 
Die Notwendigkeit kreativen Denkens ergibt sich aber nicht nur wegen der möglichen Betriebsblindheit von Experten bei der Lösung komplexer Probleme. Vielmehr erweist sich in einer Welt, in der sich bestimmte Probleme wie z.B. die Versorgung einer exponenziell wachsenden Menschheit mit Nahrung und Wasser immer drängender stellen, in der das kriegerische Zerstörungspotenzial nach wie vor zur mehrfachen Vernichtung des Globus ausreicht, und in der anthropogene Emissionen inzwischen einen erheblichen Anteil an empfindlichen natürlichen Stoffkreisläufen nehmen (vgl. WBGU 1999), das kreative Potenzial der Menschheit als ein möglicher Hoffnungsschimmer. Die gesamte (Kultur-)Geschichte der Menschheit wäre ohne kreative Prozesse nicht in der Weise verlaufen, die wir heute rekonstruieren.

Aus diesem Grund ist es wichtig, nicht nur die Bedingungen kreativer Tätigkeit zu studieren, sondern aktive Maßnahmen zur Förderung des kreativen Denkens zu ergreifen. Elternhaus, Schule und Universität stellen ja in gewissem Sinne Sozialisationsinstanzen dar, die zur Förderung kreativen Verhaltens anhalten sollten. Mit den Fördermöglichkeiten beschäftigt sich der letzte Abschnitt.

Was kann man zur Förderung kreativen Denkens tun?

Zur Förderung kreativen Denkens sind viele Programme aufgelegt und zahllose Empfehlungen ausgesprochen worden. Auch wenn es Forscher gibt, die der Ansicht sind, dass kreatives Potenzial nur wenigen Menschen vorbehalten sei, ist die Mehrzahl der Kreativitätsforscher der Meinung, dass jeder Einzelne seine Kreativität entfalten kann. Teresa Amabile (1983; vgl. auch Amabile 1996) nennt Entscheidungsfreiheit, unerwartete Bekräftigungen, positives Innovationsklima, stimulierendes Milieu als kreativitätsförderliche Faktoren, als hinderlich dagegen Druck von Kollegen sowie Druck durch Supervision und durch erwartete Evaluation.

Nach Sternberg und Lubart (1991) müssen verschiedene individuelle wie Umwelt-Faktoren zusammenkommen. Den Empfehlungen folgend, die Sternberg $(1995,363 f$.$) zur Erhöhung des kreativen Outputs empfiehlt, sollte$ man auf die folgenden Punkte achten:

1. Entwickeln Sie eine hohe Motivation dafür, auf einem speziellen Gebiet kreativ zu sein! Lassen Sie sich um keinen Preis durch extrinsische Motivation (z.B. in Form von Geld) als Entschädigung für kreative Leistungen bestechen - Geld korrumpiert! Generell sollte das Streben zu kreativen Handlungen aus Ihnen selbst kommen (intrinsische Motivation)!

2. Zeigen Sie ein gewisses Maß an Nonkonformismus - Regeln, die Ihre kreativen Handlungen beschränken, können gegebenenfalls missachtet wer- 
den! Allerdings: nicht alle Regeln und Gewohnheiten sind schädlich. Was die eigene Leistung angeht: höchste Ansprüche und Selbstdisziplin beim Schaffen sind nötig!

3. Sie müssen vom Wert und der Bedeutung Ihrer kreativen Tätigkeit völlig überzeugt sein, Kritik und Abwertung durch andere Personen darf Sie nicht stören! Die Selbstkritik sollte jedoch den eigenen Prozess überwachen und verbessern!

4. Suchen Sie sich Gegenstände und Personen, auf die sich Ihre kreative Aufmerksamkeit konzentriert, sorgfältig aus - dabei kann es sich auch (und gerade) um solche handeln, die von anderen Personen nicht geschätzt werden.

5. Benutzen Sie Analogien und divergentes Denken, wo immer möglich. Aber: kreatives Denken berücksichtigt auch die alten Traditionen - und sei es nur, um ihnen zu widersprechen.

6. Suchen Sie sich Mitstreiter, die gegen die Konvention angehen und neue Ideen ausprobieren, Mitstreiter, die zum Risiko ermutigen!

7. Sammeln Sie soviel Wissen über Ihren Bereich wie möglich! Damit kann verhindert werden, dass das Rad zum 100. Mal erfunden wird. Vermeiden Sie gleichzeitig, von diesen Daten gefesselt zu werden!

8. Verpflichten Sie sich auf das strengste zu Ihren kreativen Unternehmungen.

Wie man an diesen Empfehlungen sehen kann, wird nicht ein einzelner Faktor verantwortlich gemacht, sondern ein breites Bündel förderlicher Maßnahmen geschnürt. Neben einer kreativitätsförderlichen Umwelt müssen nötiges Wissen, entsprechende Persönlichkeitsmerkmale, intellektuelle Prozesse und auch genug intrinsische Motivation vorhanden sein.

Sternberg und Lubart $(1991,1995)$ haben ihre Vorstellungen übrigens als „Investment-Theorie der Kreativität" bezeichnet. Sie wollen damit ausdrücken: das kreative Individuum „buys low and sells high“. „Buys low“ soll dabei bedeuten: eine von den Zeitgenossen unterschätzte (und damit „billige“) Idee wird aufgegriffen und kreativ weiterentwickelt. „Sells high“ soll bedeuten: Wenn diese weiterentwickelte Idee andere Menschen überzeugt, kann sie teuer verkauft werden.

Nochmals zurück zur Förderung der Kreativität. Ein wichtiges Merkmal vieler Förderprogramme ist das Zurückstellen evaluativer Prozesse in der Phase, in der Ideen generiert werden. Damit soll ein vorschnelles Verwerfen origineller Ideen verhindert werden. Kommt die Evaluation zu früh ins Spiel, kann dies innovationshemmend sein. Rüdiger Ahrens (2000) beschreibt am Beispiel der Forschungsevaluation britischer Universitäten die durchaus negativen Folgen dieser Praxis für die Innovationskraft an den Hochschulen. 
Das Zurückstellen von Bewertungen ist zentraler Bestandteil einer Methode, die der Werbefachmann Alexander Osborn in den 5oer Jahren unter dem Namen „brainstorming“ propagiert hatte (Osborn 1953). Eine kleine Gruppe von Personen, etwa 6-8, sollte zu einem Thema für 60 Minuten Ideen generieren - Kritik und Diskussion waren während dieser Periode explizit verboten. Erst anschließend wurde die protokollierte Liste von Ideen durchgegangen und jeweils gefragt: Lässt sich diese Idee sofort umsetzen? Wie weit muss die Idee ausgebaut werden? Kann die Idee überhaupt umgesetzt werden?

Die von Osborn vorgeschlagene Trennung zwischen Produktions- und Bewertungsphase hat sich im Kontext kreativer Prozesse als äußerst erfolgreich erwiesen (vgl. Taylor 1964) und ist durch eine Reihe von Variationen ergänzt worden (vgl. Seiffge-Krenke 1974, S. 264f.). Wie Farr (1990) schreibt, ist Brainstorming nach wie vor eine sehr populäre Technik, die in vielen Unternehmen zum Einsatz kommt. Inwiefern Versuche erfolgreich sein werden, diese Technik als „electronic brainstorming“, also vermittelt über Computerinteraktion zu etablieren, wird die Zukunft zeigen (z.B. Roy, Gauvin \& Limayem 1996).

Dass Kreativität nicht nur von den Personen, sondern auch vom verfügbaren Wissen in einer Domäne abhängt, zeigt sich in der Wissenschaftsgeschichte immer wieder. Sobald bestimmte grundlegende Ideen in einer ,jungen“ Disziplin bekannt werden, kommt es zu einer explosionsartigen Zunahme an kreativen Ideen in diesem Bereich. Wenn nach einiger Zeit das Wissen stark gewachsen und die Lücken kleiner geworden sind, lassen auch die kreativen Erfindungen nach - die Domäne kommt aus dem positiv beschleunigten Ast der Entwicklung (= Wachstumsprozesse) in den negativ beschleunigten Ast (= Bremsprozesse), wo allmählich die Decke erreicht wird.

\section{Abschließende Bemerkungen}

Die hier vorgetragenen Überlegungen verdeutlichen nochmals die Notwendigkeit zu einer Perspektive, in der kreatives Denken als Interaktionsprozess zwischen einer kreativen Persönlichkeit und einer kreativitätsförderlichen Umwelt konzipiert wird. Sie verdeutlichen ebenfalls, dass kreative Leistungen nicht „verordnet“ werden können, sondern einen Schatz darstellen, zu dessen Pflege die institutionellen Bedingungen in Schulen und Universitäten sorgsam überdacht werden müssen. Gemessen an der erdrückenden Menge an Problemen, mit denen sich die Menschheit auf globaler Ebene konfrontiert sieht, ist eine große Anstrengung erforderlich, diese Kräfte auf positive Ziele zu bündeln. Gerade die Psychologie der Kreativität zeigt, dass dies nicht dem einzelnen allein überlassen werden kann. 


\section{Literatur}

Ahrens R (2000) Eine Gefahr für die Universitäten? Forschungsevaluation in Großbritannien. Forschung \& Lehre 7 (Heft 4):182-184

Amabile TM (1983) The social psychology of creativity. Springer, New York

Amabile TM (1996) Creativity in context. Westview, Boulder, CO

Amelang M, Bartussek D (1997) Differentielle Psychologie und Persönlichkeitsforschung. Vierte, überarbeitete und erweiterte Auflage. Kohlhammer, Stuttgart

Boden MA (1991) The creative mind: Myths \& mechanisms. Basic Books, New York

Bredenkamp J (1980) Theorie und Planung psychologischer Experimente. Steinkopff, Darmstadt

Csikszentmihalyi M (1997) Creativity: Flow and the psychology of discovery and invention. Harper Collins, New York

Csikszentmihalyi M (1999) Creativity. In: Wilson RA, Keil FC (eds) The MIT encyclopedia of the cognitive sciences. MIT Press, Cambridge, MA, pp 205-206

Dorfman J, Shames VA, Kihlstrom JF (1996) Intuition, incubation, and insight: Implicit cognition in problem solving. In: Underwood G (ed), Implicit cognition. Oxford University Press, Oxford, pp 257-296

Ericsson KA (1996) (ed) The road to expert performance: Empirical evidence from the arts and sciences, sports, and games. Mahwah, NJ: Erlbaum

Eysenck HJ (1995) Genius: The natural history of creativity. Cambridge University Press, Cambridge

Farr JL (1990) Facilitating individual role innovation. In: West MA, Farr JL (eds) Innovation and creativity at work: Psychological and organizational strategies. Wiley, New York, pp 207-230

Finke RA, Ward TB, Smith SM (1992) Creative cognition: Theory, research, and applications. MIT Press, Cambridge, MA

Frensch PA, Sternberg RJ (1989) Expertise and intelligent thinking: When is it worse to know better? In: Sternberg RJ (ed), Advances in the psychology of human intelligence. Lawrence Erlbaum, Hillsdale, NJ, pp 157-188

Funke J (1999) (Hrsg) Komplexes Problemlösen [Themenheft]. Psychologische Rundschau 50(4)

Galton F (1869) Hereditary genius: An inquiry into its laws and consequences. Macmillan, London

Gardner H (1983) Frames of mind: A theory of multiple intelligences. Basic Books, New York

Gardner H (1993) Creating minds: An anatomy of creativity seen through the lives of Freud, Einstein, Picasso, Stravinsky, Eliot, Graham and Gandhi. Basic Books, New York

Gentner D, Gentner DR (1983) Flowing waters and teeming crowds: Mental models of electricity. In: Gentner D, Stevens AL (eds) Mental models. Lawrence Erlbaum, Hillsdale, NJ, pp 99-130

Getzels JW, Jackson P (1962) Creativity and intelligence. Wiley, New York

Guilford JP (1950) Creativity. American Psychologist 5:444-454

Guilford JP (1967) The nature of human intelligence. McGraw-Hill, New York

Hocevar D, Bachelor P (1989) A taxonomy and critique of measurement used in the study of creativity. In: Glover JA, Ronning RR, Reynolds CR (eds) Handbook of creativity. Plenum Press, New York, pp 53-76

Holyoak KJ (1985) The pragmatics of analogical transfer. The Psychology of Learning and Motivation 19:59-87

Hussy W (1986) Denkpsychologie. Ein Lehrbuch. Band 2: Schlußfolgern, Urteilen, Kreativität, Sprache, Entwicklung, Aufmerksamkeit. Kohlhammer, Stuttgart

Kämmerer A (2000) Kreativität und Geschlecht oder „Not macht erfinderisch“. (in diesem Band) 
Krampen G (1993) Diagnostik der Kreativität. In: Trost G, Ingenkamp K, Jäger RS (Hrsg) Tests und Trends. 10. Jahrbuch der Pädagogischen Diagnostik. Beltz, Weinheim, pp 11-39

Krems JF (1995) Cognitive flexibility and complex problem solving. In: Frensch PA, Funke J (eds) Complex problem solving: The European perspective. Lawrence Erlbaum Associates, Hillsdale, NJ, pp 201-218

Lindauer MS (1993) The span of creativity among long-lived historical artists. Creativity Research Journal 6:231-239

Lubart TI (1994) Creativity. In: Sternberg RJ (ed) Thinking and problem solving. Academic Press, San Diego, CA, pp 290-323

Luchins AS (1942) Mechanization in problem solving. Psychological Monographs 54

Ludwig AM (1995) The price of greatness: Resolving the creativity and madness controversy. Guilford Press, New York

Martindale C (1989) Personality, situation, and creativity. In: Glover JA, Ronning RR, Reynolds CR (eds) Handbook of creativity. Plenum Press, New York, pp 211-232

Martindale C (1990) The clockwork muse: The predictability of artistic styles. Basic Books, New York

Mednick SA (1962) The associative basis of the creative process. Psychological Review 69: 220-232

Osborn AF (1953) Applied imagination. Scribner's, New York

Rothenberg A (1990) Creativity and madness: New findings and old stereotypes. John Hopkins University Press, Baltimore

Roy MC, Gauvin S, Limayem M (1996) Electronic group brainstorming: The role of feedback on productivity. Small Group Research 27:215-247

Seiffge-Krenke I (1974) Probleme und Ergebnisse der Kreativitätsforschung. Huber, Bern

Simonton DK (1984) Genius, creativity, and leadership: Historiometric inquiries. Harvard University Press, Cambridge, MA

Simonton DK (1994) Greatness: Who makes history and why. Guilford Press, New York

Simonton DK (1997) Creative productivity: A predictive and explanatory model of career trajectories and landmarks. Psychological Review 104:66-89

Simonton DK (1999) Origins of genius: Darwinian perspective on creativity. Oxford University Press, New York

Simonton DK (2000) Creativity. Cognitive, personal, developmental, and social aspects. American Psychologist 55:151-158

Smith SM, Ward TB, Finke RA (1995) (eds) The creative cognition approach. MIT Press, Cambridge, MA

Sternberg RJ (1995) In search of the human mind. Harcourt Brace College Publishers, Fort Worth, TX

Sternberg RJ, Lubart TI (1991) An investment theory of creativity and its development. Human Development 34:1-31

Sternberg RJ, Lubart TI (1995) Defying the crowd: Cultivating creativity in a culture of conformity. Free Press, New York

Subotnik RF, Arnold KD (1994) (eds) Beyond Terman: Contemporary longitudinal studies of giftedness and talent. Ablex, Norwood, NJ

Taylor CW (1964) Creativity: Progress and Potential. McGraw-Hill, New York

Terman RM (1925) Mental and physical traits of a thousand gifted children. Stanford University Press, Stanford, CA

Torrance EP (1966) Torrance Test of Creative Thinking. Directions manual, and scoring guide. Personnel Press, Princetown, NJ

Wallas G (1926) The art of thought. Harcourt Brace, New York

Ward TB, Smith SM, Vaid J (1997) (eds) Creative thought: An investigation of conceptual structures and processes. American Psychological Association, Washington, DC 
WBGU [= Wissenschaftlicher Beirat Globale Umweltveränderungen] (1999) Welt im Wandel: Strategien zur Bewältigung globaler Umweltrisiken. Jahresgutachten 1998. Springer, Heidelberg

Weisberg RW (1989) Kreativität und Begabung. Was wir mit Mozart, Einstein und Picasso gemeinsam haben. Spektrum der Wissenschaft Verlagsgesellschaft, Heidelberg

\section{Abbildungsnachweis}

[1] The Sculpture Center, Ohio/USA; Foto: Archiv

[2] Torrance 1966; aus Hussy 1986, S. 76; Abdruck mit Genehmigung des Kohlhammer-Verlags

[3] design \& production $\mathrm{GmbH}$, Heidelberg 2000

Für wertvolle Hinweise zu einer Vorfassung dieses Beitrags danke ich Frau Dr. Marlene Endepohls, Handschuhsheim. 